\title{
ДИАГНОСТИКА ИНФЕКЦИЙ, ПЕРЕДАЮЩИХСЯ ПОЛОВЫМ ПУТЕМ ПРИ ПОМОЩИ ПЬЕЗОКВАРЦЕВОГО МИКРОВЗВЕШИВАНИЯ
}

\section{DIAGNOSIS OF SEXUALLY TRANSMITTED INFECTIONS BY USING QUARTZ CRYSTAL MICROBALANCE METHOD}

\section{A. Smetankina}

Summary. Diagnostic measures to detect sexually transmitted infections (STIs) require an individual approach. The aim of the study was to study the possibilities of using the piezoelectric quartz weighing method in the diagnosis of STls using solid sorbent films. We analyzed 30 samples of cervical mucus, divided into 2 groups: 20 samples - a training sample and 10 samples - a test sample. As a result, a method was developed for measuring the smell of a biological sample (smear of cervical mucus of the cervix uteri) with frontal input of vapors, quantitative recorded and calculated parameters for ranking samples into groups were established. Testing results of the developed approach were evaluated by gynecologists as practically important.

Keywords: bioprobes, artificial systems, piezo-quartz microweaving, artificial intelligence, multisensory systems.

\section{Введение}

овременная клиническая картина инфекций, передаваемых половым путем (ИПП), характеризуется стертой симптоматикой и рецидивирующим течением, что обусловлено возникновением новых резистентных штаммов возбудителей. Соответственно, актуальность ранней лабораторной диагностики ИППП не вызывает сомнения. В настоящее время существуют различные методы диагностики половых инфекций, которые различаются между собой чувствительностью, удобством применения, общедоступностью и ценой. Опыт лечения заболеваний, передающихся половым путем, показывает, что назначение диагностических мероприятий требует индивидуального подхода

\section{Le^ь}

Использование метода пьезокварцевого микровзвешивания в диагностике инфекций, передающихся половым путем, с использованием твёрдых пленок сорбентов.

\author{
Сметанкина Анастасия Васильевна \\ Аспирант, Воронежский государственный \\ медицинский университет им. Н. Н. Бурденко \\ Zhuchkova954@yandex.ru
}

Аннотация. Диагностические мероприятия для выявления инфекций, передающихся половым путем (ИППП), требуют индивидуального подхода. Целью исследования стало изучение возможностей использования метода пьезокварцевого микровзвешивания в диагностике ИППП, с использованием твёрдых пленок сорбентов. Проанализировано 30 проб цервикальной слизи, разделенных на 2 группы: 20 проб - обучающая выборка и 10 проб — проверочная выборка. В результате был разработан способ измерения запаха биопробы (мазок цервикальной слизи шейки матки) с фронтальным вводом паров, установлены количественные регистрируемые и расчетные параметры ранжирования проб на группы. Результаты апробации разработанного подхода оценены врачами гинекологами, как практически важные.

Ключевые слова: биопробы, AquaStok, пьезокварцевое микровзвешивание, искусственный интеллект, мультисенсорные системы.

\section{Материа^ и методы}

В исследование были включены 30 пациенток у которых не было как клинических проявлений, так и жалоб в целом. Проанализировано 30 проб цервикальной слизи, которые разделены на 2 выборки: 20 проб - обучающая выборка и 10 проб - проверочная выборка. На основании предварительного диагноза, пробы из обучающей выборки были разделены на группы: «клинически здоровые», «инфекция». Всем пациенткам с диагностической целью был применен традиционный метод обследования (ПЦР, Фемофлор), а также метод пьезокварцевого микровзвешивания (ПКМ).

\section{Результаты \\ и обсужАение}

Первым значимым отличительным параметром является $\mathrm{S}_{6 . o}$ (площадь визуального отпечатка). Установлено, что наименьшая $\mathrm{S}_{6 . o}$ характерна для группы «клинически здоровые», существенно ниже значений для группы «инфекция» т.е. при воспроизводимом от- 
Таблица 1. Соотношение сигналов нескольких сенсоров в матрице для тестируемых проб $( \pm 0,02)$

\begin{tabular}{|c|c|c|c|c|c|c|c|c|c|c|c|c|c|}
\hline \multirow{2}{*}{ № пробы } & \multicolumn{13}{|l|}{$\boldsymbol{A}_{i / j}$} \\
\hline & $1 / 2$ & $1 / 7$ & $2 / 3$ & $3 / 4$ & $3 / 5$ & $2 / 6$ & $2 / 7$ & $1 / 5$ & $5 / 6$ & $5 / 7$ & $4 / 5$ & $4 / 6$ & $4 / 7$ \\
\hline & 1 & 2 & 3 & 4 & 5 & 6 & 7 & 8 & 9 & 10 & 11 & 12 & 13 \\
\hline № 1 & 2 & 1 & 0,13 & 1,33 & 8 & 1 & 0.5 & 2 & 1 & 0,5 & 6 & 6 & 3 \\
\hline № 2 & 2 & 2 & 0,20 & 1,25 & 5 & 1 & 1 & 2 & 1 & 1 & 4 & 4 & 4 \\
\hline № 3 & 0,5 & 1 & 0,20 & 1,67 & 10 & 1 & 2 & 1 & 0,5 & 1 & 6 & 3 & 6 \\
\hline № 4 & 2 & 2 & 0,20 & 2,5 & 5 & 1 & 1 & 2 & 1 & 1 & 2 & 2 & 2 \\
\hline № 5 & 2 & 1 & 0,13 & 1,6 & 4 & 0,5 & 0.5 & 2 & 0,5 & 0,5 & 5 & 2,5 & 2,5 \\
\hline № 6 & 0,33 & 0,26 & 0,08 & 2,11 & 11,9 & 0,75 & 0.78 & 0.34 & 0,73 & 0,76 & 5,66 & 4,13 & 4,3 \\
\hline № 7 & 0,38 & 0,29 & 0,10 & 1,68 & 8,1 & 0,81 & 0.75 & 0.31 & 0,98 & 0,91 & 4,82 & 4,73 & 4,39 \\
\hline № 8 & 0,38 & 0,25 & 0,10 & 1,64 & 6,71 & 0,70 & 0.66 & 0.27 & 0,98 & 0,93 & 4,10 & 4,03 & 3,79 \\
\hline № 9 & 0,26 & 0,11 & 0,11 & 1,36 & 4,34 & 0,65 & 0.42 & 0.12 & 1,36 & 0,87 & 3,19 & 4,35 & 2,79 \\
\hline № 10 & 0,30 & 0,13 & 0,11 & 1,36 & 4,37 & 0,64 & 0.44 & 0.15 & 1,29 & 0,89 & 3,22 & 4,15 & 2,85 \\
\hline \multicolumn{14}{|c|}{ Среднее } \\
\hline № 11 & 0,28 & 0,14 & 0,13 & 1,14 & 3,52 & 0,61 & 0.49 & 0.13 & 1,29 & 1,03 & 3,08 & 3,98 & 3,17 \\
\hline № 12 & 0,31 & 0,20 & 0,12 & 1,31 & 4,49 & 0,78 & 0.65 & 0.17 & 1,41 & 1,01 & 3,41 & 4,83 & 4,06 \\
\hline № 13 & 0,24 & 0,16 & 0,21 & 1,02 & 2,4 & 0,82 & 0.67 & 0.12 & 1,61 & 1,32 & 2,35 & 3,78 & 3,1 \\
\hline № 14 & 0,31 & 0,20 & 0,13 & 1,25 & 4,31 & 0,83 & 0.64 & 0.18 & 1,48 & 1,14 & 3,46 & 5,13 & 3,96 \\
\hline № 15 & 0,39 & 0,21 & 0,09 & 1,72 & 5,66 & 0,66 & 0.53 & 0.21 & 1,22 & 0,99 & 3,28 & 4,0 & 3,24 \\
\hline № 16 & 0,33 & 0,17 & 0,10 & 1,81 & 6,11 & 0,63 & 0.52 & 0.20 & 1,07 & 0,88 & 3,37 & 3,60 & 2,98 \\
\hline № 17 & 0,35 & 0,22 & 0,10 & 1,58 & 6,0 & 0,79 & 0.63 & 0.21 & 1,27 & 1,02 & 3,81 & 4,85 & 3,9 \\
\hline № 18 & 0,36 & 0,22 & 0,09 & 1,79 & 6,03 & 0,71 & 0.60 & 0.20 & 1,29 & 1,08 & 3,38 & 4,35 & 3,65 \\
\hline № 19 & 0,40 & 0,24 & 0,10 & 1,65 & 5,57 & 0,71 & 0.61 & 0.21 & 1,34 & 1,15 & 3,38 & 4,54 & 3,88 \\
\hline № 20 & 0,38 & 0,20 & 0,08 & 1,70 & 6,24 & 0,62 & 0.53 & 0.20 & 1,15 & 1,0 & 3,67 & 4,23 & 3,67 \\
\hline
\end{tabular}

* отмечен параметр с максимальным отклонением от стандартов (контроля).

боре массы проб и соблюдении времени между отбором пробы и проведением измерения наиболее значимым, и простым параметром для прогнозирования диагноза является $\mathrm{S}_{6.0}$. Так как на этот параметр сильно влияют условия отбора, то необходимо проанализировать другие возможные параметры ранжирования для проб.

Параметр $A_{i / j i}$ отражает постоянство соотношения концентраций отдельных классов легколетучих соединений в РГФ (табл. 2). Если показатели $A_{i j j}$ для проб близки или совпадают, то можно считать, что соотношение содержания в пробах отдельных соединений или классов одинаково. Если соотношение сигналов отличается от таких для проб, то соотношение концентрацией этих групп соединений различно, по сравнению с соответствующим стандартом или средней выборкой стандартов (таблица 7). Установлено, что не все параметры $A_{i j}$ для выбранных групп проб различаются. В таблице 1 выделены показатели $A(3 / 4), A(3 / 5), A(2 / 7), A(1 / 5), A(5 / 6)$, значения которых отражают изменения состава летучих соединений для проб.

Чем больше число параметров $\mathrm{A}_{i j}$ для групп «инфекция», «пролеченные», отличающихся от группы «клинически здоровые», тем существеннее отличия по запаху проб, которые с высокой степенью вероятности отражают наличие и интенсивность протекания патогенных процессов в организме (таблица 2).

Полученные круговые спектры идентификационных параметров были построены как спектры, которые позволяют отнести пробу к определенной группе. Так как химический состав пробы «клинически здоровых» принципиально отличается от проб «инфекция», поэтому их форма спектра имеет значимые различия. На основании 
Таблица 2. Качественные характеристики проб для ИППП

\begin{tabular}{|l|l|}
\hline Диагноз & Запах \\
\hline Бактериальный вагиноз & Аммиак, амины, запах тухлой рыбы \\
\hline ВПЧ & Запах тухлой рыбы \\
\hline Кандидоз & Аммиак, кислый запах \\
\hline Хламидиоз & $\begin{array}{l}\text { Аминокислоты, амины (неприятный } \\
\text { рыбный запах) }\end{array}$ \\
\hline Уреаплазмоз & Нет специфического запаха \\
\hline Микоплазмоз & Запах нашатыря \\
\hline
\end{tabular}

Таблица 3. Минимально достаточный объем информации пьезокварцевого микровзвешивания массивом сенсоров на наноматериалах для ранжирования состояния гинекологических биопроб

\begin{tabular}{|c|c|c|}
\hline Регистрируемые отклики & $\begin{array}{l}\text { По форме круговых спектров } \\
\text { Подобие фигурам }\end{array}$ & Диагностические группы \\
\hline \multicolumn{3}{|l|}{$\mathrm{S}_{6.0,}$, Гц्С } \\
\hline $100 \pm 10$ & 6 & «Клинически здоровые» \\
\hline \multicolumn{3}{|l|}{$\mathrm{S}_{6.0}, \Gamma Ц \mathrm{C}^{\circ} \mathrm{C}$} \\
\hline Выше 30000 & 3 & $\begin{array}{l}\text { «Микст-активные от- клонения»: } \\
\text { «Инфекция» }\end{array}$ \\
\hline$(5000-29500) \pm 500$ & 8 & \\
\hline
\end{tabular}

формы спектра, которая соответствует каждой группе, можно разделить все пробы из обучающей выборки.

Наиболее простой алгоритм получения дополнительной диагностической информации предлагаемым способом представлен в таблице 3, параметры расположены в порядке уменьшения значимости, но анализируются в совокупности. Если присутствует хотя бы один параметр, это является основанием помещения группы в определенную группу. При этом отклонение остальных показателей от указанных для этих групп значений, свидетельствует о наличии не активных, но присутствующих изменений, отличных от нормы

Для поиска корреляций с более детальными диагнозами (идентификация типа возбудителя или вида воспа- ления) необходимо существенное увеличение объема выборки с индивидуальными и микст-инфекциями.

Разработан способ измерения запаха биопробы (мазок цервикальной слизи шейки матки) на приборе «AquaStok» с фронтальным вводом паров, установлены количественные регистрируемые и расчетные параметры ранжирования проб на группы. Результаты апробации разработанного подхода оценены врачами гинекологами, как практически важные.

Аналитические характеристики разработанного способа перспективны для быстрой оценки репродуктивного статуса пациенток, грамотного подбора антибиотикотерапии, профилактики и уменьшения количества органоуносящих операций.

\section{ЛИТЕРАТУРА}

1. Гинекология. Национальное руководство./ под редакцией Кулакова В. И., Савельева Г. М., Манухина И. Б. — Издательская группа « ГЭОТАР — Медиа», 2009-С. 356

2. Венерические болезни. Руководство для врачей./ под ред. О. К. Шапошникова.- М., Медицина, 1991 — С. 413-430 
3. Pavlou A. K., Magan N., McNulty C., Jones J. M., Sharp D., Brown J., Turner A. P.F. Use of an electronic nose system for diagnoses of urinary tract infections// Biosens. Bioelectron. 2002. V. 17. № 10. P. 893.

4. Bernabei M., Pennazza G., Santonico M., Corsi C., Roscioni C., Paolesse R., Di Natale C., D'Amico A. A preliminary study on the possibility to diagnose urinary tract cancers by an electronic nose // Sens. Actuators B: Chem. 2008. V. 131. № 1. P. 1.

5. Бельский В. Е. Солюбилизация газов и насыщенных паров в биологических средах // Хим.-фарм. журнал. 2000. Т. 34. № 11. С. 42.

6. Buszewski B., Ulanowska A., Kowalkowski T., CieslinskiK. Identification of volatile lung cancer markers by gas chromatography-mass spectrometry: Comparison with discrimination by canines // Anal. Bioanal. Chem. 2012. V. 404. № 1-2. P. 141.

7. Gaspar E. M., Lucena A. F., Duro da Costa J., Chavesdas Neves H. Organic metabolites in exhaled human breath — A multivariate approach for identification of biomarkers in lung disorders // J. Chromatogr. A. 2009. V. 1216. № 14. P. 2749.

8. Gardner J. W., Shin H.W., Hines E. L. An electronic nose system to diagnose illness // Sens. Actuators B: Chem. 2000. V. 70. № 1-3. P. 19.

9. Кучменко Т. А., Шуба А. А., Тюркин И.А, Битюкова В. В. Оценка состояния биологических проб по составу равновесной газовой фазы с примене нием мультисенсорной системы // Журн. аналит. химии. 2014. Т. 69. № 5. С. 534.

10. Weiss T., Angerer J. Simultaneous determination of various aromatic amines and metabolites of aromatic nitro compounds in urine for low level exposure using gas chromatography-mass spectrometry // J. Chromatogr. B. 2002. V. 778. № 1-2.P. 179.

11. Deng Y.-H., Wang H., Zhong L., Zhang H.-S. Trace determination of short-chain aliphatic amines in biological samples by micellar electrokinetic capillary chromatography with laser-induced fluorescence detection // Talanta. 2009. V. 77. № 4. P. 1337.

12. Rudnicka J., Kowalkowski T., Ligor T., Buszewski B. Determination of volatile organic compounds as biomarkers of lung cancer by SPME-GC-TOF/MS and chemometrics // J. Chromatogr. B. 2011. V. 879. № 30. P. 3360.

13. Senthilmohan S. T., Kettle A. J., McEwan M.J, Dummer J., Edwards S. J., Wilson P.F., Epton M. J. Detection of monobromamine, monochloramine and dichloramine using selected ion flow tube mass spectrometry and their relevance as breath markers // Rapid Commun. Mass Spectrom. 2008. V. 22. № 5. P. 677.

14. Carrola J, Rocha C. M., Barros A. S., Gil A. M., Goodfellow B. J., Carreira I. M., Bernardo J., Gomes A., Sousa V., Carvalho L., Duarte I. F. Metabolic signatures of lung cancer in biofluids: NMR-based metabonomics of urine // J. Proteome Res. 2011. V. 10. № 1. P. 221.

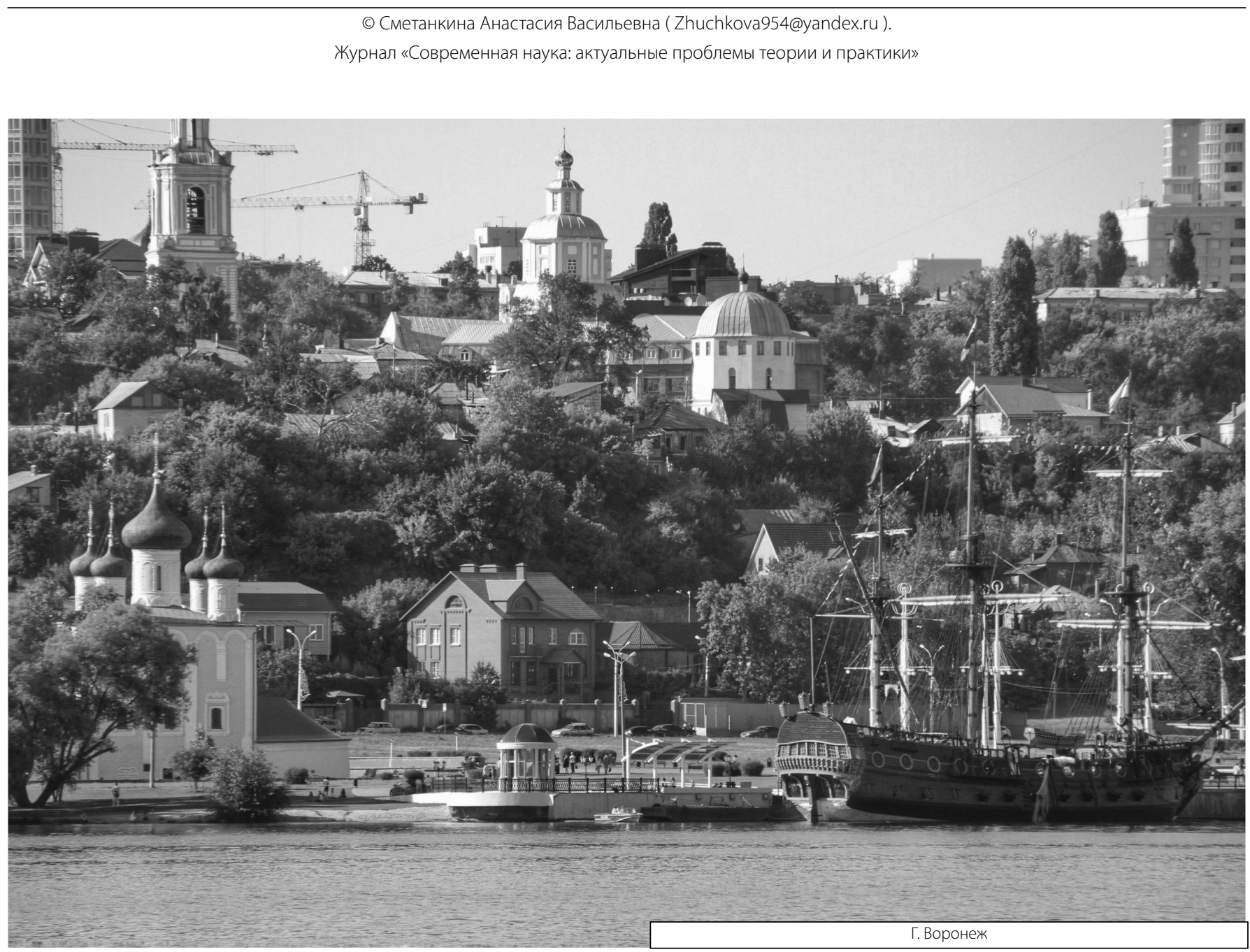

